\title{
Comunicação
}

[Communication]

\section{Aspectos parasitológicos da traíra (Hoplias malabaricus) proveniente da cidade de São Bento, MA}

[Parasitologic aspects of traíra (Hoplias malabaricus) from the São Bento city, MA]

\author{
L.C. Rodrigues ${ }^{1}$, A.C.G. Santos ${ }^{2}$, E.M. Ferreira ${ }^{2}$, T.S. Teófilo ${ }^{2}$, D.M. Pereira ${ }^{2}$, F.N. Costa ${ }^{2} *$ \\ ${ }^{1}$ Instituto Federal de Educação, Ciência e Tecnologia do Maranhão, Zé Doca, MA \\ ${ }^{2}$ Universidade Estadual do Maranhão - UEMA,São Luís, MA
}

Parasitos podem ser patogênicos para os peixes, pois costumam invadir órgãos como fígado, gônadas, mesentério e musculatura corporal, provocando extensa patologia, principalmente quando um grande número está presente (Okumura et al., 1999).

Além dos danos diretos proporcionados aos peixes e das perdas econômicas, as zoonoses parasitárias veiculadas por pescado cada vez mais vêm chamando a atenção de pesquisadores e autoridades sanitárias, por serem causas de problemas na população, que pode ser infectada, pelo consumo de pescado cru ou cozido insuficientemente (Okumura et al., 1999).

Dentre as espécies de peixes nativas provenientes das regiões alagadas da Baixada Maranhense, destaca-se a Hoplias malabaricus (Bloch, 1794), conhecida popularmente como "traíra", um peixe carnívoro, essencialmente piscívoro, pertencente à família Erythrinidae, que possui ampla distribuição na América do Sul, exceto na região dos Andes e nos rios da Patagônia (Nakatani et al., 2001). Tendo em vista a importância da atividade pesqueira artesanal nessa região, buscou-se avaliar as condições higiênicosanitárias da traíra (Hoplias malabaricus), provenientes do município de São Bento-MA, quanto a seus aspectos parasitológicos.

O estudo foi desenvolvido no município de São

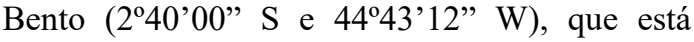
localizado na mesorregião Norte Maranhense e na microrregião Baixada Maranhense (Maranhão

Recebido em 6 de outubro de 2015

Aceito em 12 de maio de 2016

*Autor para correspondência (corresponding author)

E-mail: francisca.cca.uema@gmail.com
- Brasil). O clima da região, segundo a classificação de Thorntwaite (1948), é úmido. Apresenta temperatura média anual superior a $27^{\circ} \mathrm{C}$, umidade relativa do ar anual superior a $82 \%$ e precipitação pluviométrica anual com totais entre 1600 e $2000 \mathrm{~mm}$ (Atlas..., 2002).

Foram utilizados 70 peixes da espécie $H$. malabaricus (traíra), de ambos os sexos, coletados no período de outubro de 2010 a janeiro de 2011. As traíras (H. malabaricus) foram coletadas em locais de captura e feiras livres, diretamente do pescador. Os espécimes foram separados em sacos de polietileno estéreis, identificados e acondicionados em caixas isotérmicas e, em seguida, encaminhados para análises parasitológicas.

Foi realizada a inspeção macroscópica do tegumento com auxílio de lupa manual. Logo após, realizaram-se a remoção do opérculo para análise das brânquias e dos arcos branquiais, a abertura da cavidade visceral e a exposição dos órgãos. Em seguida, estes foram retirados e separados em placas de Petri contendo água destilada e, depois, examinados ao esteriomicroscópio.

Os parasitos foram coletados por órgãos, colocados em placas de Petri, lavados em água destilada, quantificados, separados e transferidos para frascos de vidro contendo solução conservante de AFA. Posteriormente, foram clarificados em lactofenol de Amann por meio da técnica de montagem rápida (Amato et al., 1991), observados entre lâminas e lamínulas para 
identificação em microscópio óptico (Zeiss) $(100 x, 400 x)$ acoplado à ocular micrométrica (25x) e para registro microfotográfico (200x). Após identificação, a conservação dos nematódeos foi feita em álcool $70^{\circ} \mathrm{GL}$ com 5$10 \%$ de glicerina.

Os nematódeos e os monogenéticos obtidos nas amostras de $H$. malabaricus foram do gênero Contracaecum spp., Pseudoterranova spp., Eustrongylides spp., Gyrodactirus spp., Dactylogirus spp. e larva Acanthor de Acanthocephalas, totalizando 1290 parasitos identificados.

Os $H$. malabaricus examinados apresentaram peso médio de $97 \pm 43$,4; média de comprimento total de $20,7 \mathrm{~cm} \pm 2,23 \mathrm{~cm}$; média de comprimento parcial de 16,1 cm $\pm 43,4$. Martins et al. (2009), ao estudar a sazonalidade de larvas de
Eustrongylides spp. em $H$. malabaricus do rio Paraná, sudeste do Brasil, observou que a alta prevalência foi entre peixes com 425-550g e $800-1.650 \mathrm{~g}$. Nesse estudo, foi atestado que o tamanho dos peixes influencia, quando considerada a prevalência de Eustrongylides.

Os nematódeos dos gêneros Anisakis $e$ Eustrongylides estavam na cavidade interna dos peixes, encistados ao mesentério. Não foram observadas alterações em estruturas internas, como fígado, coração e musculatura. A alta concentração de nematódeos foi encontrada em região próxima aos cecos pilóricos (Fig. 1 A-C). Os monogenéticos concentraram-se principalmente na porção anterior do intestino, diferindo dos acanthocephalos, que foram encontrados, em sua maioria, aderidos aos nematódeos (Fig. 1D-E).

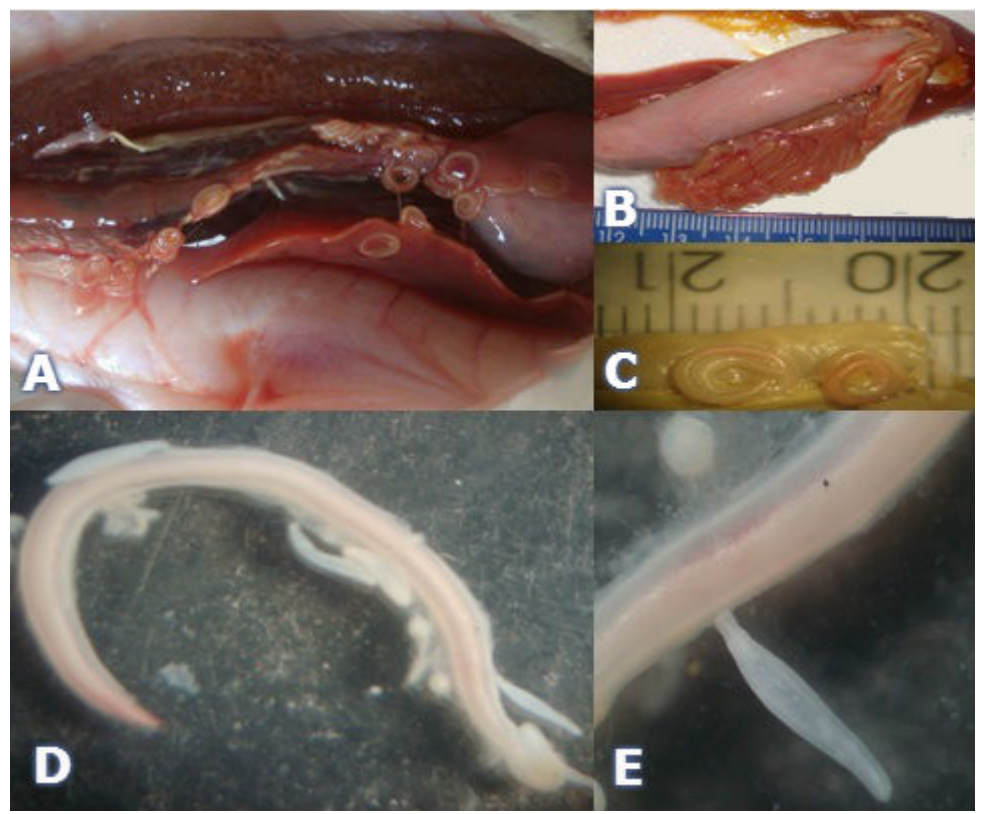

Figura 1. Presença de nematódeos Anisakidae e de classe monogenea em Hoplias malabaricus: A) nematódeos encistados no mesentério; B) nematódeos em cecos pilóricos; C) anisakídeos encistados em mesentério; D) monogenéticos encistados em nematódeos anisakídeos; E) acanthocephalo encistado em anisakídeos.

Outro nematoide encontrado em H. malabaricus foi do gênero Pseudoterranova spp., assim como em trabalhos realizados por Luque e Poulin (2004), no litoral do estado do Rio de Janeiro, os quais relataram o parasitismo de peixes marinhos por larvas de Pseudoterranova spp.
Verificou-se a presença de Eustrongylides spp. encistados no mesentério da cavidade abdominal, mas é comum a presença em fase adulta, quando o período de encistamento foi encerrado na fase de transferência de hospedeiros (peixes), penetrando nos órgãos vitais e na musculatura e, assim, habitando no corpo do animal, como verificado nos achados de necrópsia. 
Segundo Eiras (1994), a patogenia provocada pelos monogenéticos é variável com as espécies e com o local de fixação. Quando estes são fixados ao tegumento, ocorrem lesões de gravidade pouco acentuada, variável com a espécie, podendo verificar-se necrose das células, destruição de escamas e hipersecreção de muco.

Foi observada grande quantidade de girodactilídeos e dactilogirídeos na cavidade interna do peixe. Esse fato pode ser atribuído à alta densidade populacional desses parasitos no ambiente aquático, aliada ao hábito alimentar de H. malabaricus, que é um peixe piscívoro, favorecendo, dessa forma, a ingestão de ectoparasitos, assim como as adversidades do ambiente (como poluição), que contribuem para o estresse animal e, consequentemente, uma queda na imunidade dele.

Os girodactilídeos provocam uma série de reações fisiológicas no hospedeiro, como hiperplasia do tecido branquial, excessiva produção de muco e fusão dos filamentos branquiais, o que, dependendo do grau de severidade, pode levar o hospedeiro à morte por asfixia (Pavanelli et al., 2008). Eiras (1994) considerou o gênero Dactylogyrus como o mais frequente da família Dactylogyridae.

Do total de peixes examinados, 91,4\% apresentaram parasitismo por larvas de nematódeos, identificadas como Contacaecum spp., Pseudoterranova spp., Eustrongylides spp., larva de acanthella (acantocephala) e monogenoides (Dactylogyrus sp. e Gyrodactylus sp.). Dentre os peixes examinados, observou-se prevalência de $82,8 \%$ com o nematódeo Contracaecum spp., 65,7\%; Pseudoterranova spp., 7,1\%; Eustrongylides spp., 71,4\%; monogenéticos e larva acanthella de acanthocephalos, respectivamente.

As traíras parasitadas apresentaram larvas no mesentério, com exceção de um exemplar que estava inserido na bexiga natatória. A intensidade média total da infecção foi de 20,1 parasito/peixe. Os valores de amplitude de intensidade, intensidade média, índice de abundância, coeficiente de dominância, coeficiente de variação, assim como o local de infecção de larva/peixe estão apresentados na Tab. 1.

Tabela 1. Amplitude de intensidade, intensidade média, índice de abundância, coeficiente de dominância, coeficiente de variação e local de infecção dos parasitos de Hoplias malabaricus, provenientes de São Bento, MA

\begin{tabular}{lcccccc}
\hline Parasitos & A.I* & I.M* & I.A* & CD $^{*}$ & CV $(\%)^{*}$ & Local de infecção \\
\hline Contracaecum spp. & $1-27$ & 7,3 & 6,7 & 36,4 & 6,7 & mesentério \\
Pseudoterranova spp. & $1-30$ & 4,3 & 3,9 & 21,5 & 4,08 & mesentério \\
Eustrongylus spp. & $0-1$ & 0,078 & 0,071 & 0,38 & 0,07 & mesentério \\
Acanthocephala & - & 1,5 & 1,3 & 7,5 & - & mesentério \\
Gyrodactylus spp. & - & 3,7 & 3,3 & 18,3 & - & mesentério \\
Dactylogyrus spp. & - & 3,2 & 2,9 & 16 & - & mesentério \\
\hline
\end{tabular}

*Amplitude de intensidade (AI), intensidade média (IM), índice de abundância (IA), coeficiente de dominância (CD), coeficiente de Variação (CV \%)

Entre os nematódeos identificados, a maior e a menor amplitude de abundância foram verificadas para o anisakídeos, do gênero Pseudoterranova, com variação de um - 30 parasitos/peixe, seguido de Eustrongylides, com um parasito/peixe, respectivamente. Os índices mais elevados de intensidade média, coeficiente de dominância e coeficiente de variação foram para o gênero Contracaecum, seguido dos monogenéticos Gyrodactylus spp., Dactylogyrus spp. e larva de acanthocephala.
Barros et al. (2009), ao estudarem a ocorrência de endoparasitos em peixes consumidos no estado do Pará, dentre eles $H$. malabaricus, constataram que a maior prevalência e de intensidade média de parasitismo foi de helmintos da família Anisakidae encontrados em cavidade abdominal. Estes achados corraboram o presente estudo.

A presença de acanthocephala em peixes pode ser decorrente da ingestão de ostracodos (pequenos crustáceos), que contêm acanthellas completamente desenvolvidas. No trato 
digestório de peixes, os ostracodos são ingeridos, liberando as acanthellas que vão penetrar na parede do intestino, e as que já são infectantes vão alojar-se no fígado, sendo recobertas por material grandular em forma de cistos (Olsen, 1977).

Os parasitos identificados no presente estudo em $H$. malabaricus foram observados encistados no mesentério da cavidade abdominal, com alta concentração em ceco pilórico, semelhante aos achados de Muller et al. (2004), que, ao estudarem traíras provenientes de São Paulo, verificaram larvas de Contracaecum spp. no fígado e no ceco pilórico e larvas de Eustrongylides spp. na musculatura e na cavidade geral. Os endoparasitos foram os principais componentes da fauna parasitária de H.malabaricus, coincidindo com o trabalhos realizado por Martins et al. (2009).

Considerando os monogenéticos Gyrodactylus spp. e Dactylogyrus spp., foram identificados no terço final do trato digestório, aderidos ao mesentério ou fixos em outros anisakídeos, diferindo de sua característica habitual como ectoparasitos de brânquias em peixes. Verificouse nesse estudo que o coeficiente de dominância de 18,3 e 16, para Gyrodactylus spp. e Dactylogirus, respectivamente. A porcentagem de infecção aumenta de acordo com a idade/tamanho dos peixes (Tab. 2).

Tabela 2. Relação entre o comprimento total e a quantidade de nematódeos presentes em traíras (Hoplias malabaricus), provenientes do município de São Bento-MA

\begin{tabular}{lccccc} 
& \multicolumn{4}{c}{ Comprimento total $(\mathrm{cm})$} \\
& $15-17 \mathrm{~cm}$ & $18-20 \mathrm{~cm}$ & $21-23 \mathrm{~cm}$ & $24-26 \mathrm{~cm}$ & $27-30 \mathrm{~cm}$ \\
\hline $\mathrm{N}^{\text {o de peixes }}$ & 8 & 30 & 19 & 11 & 2 \\
$\mathrm{~N}^{\mathrm{o}}$ de nematódeos & 21 & 256 & 210 & 190 & 76 \\
Média (parasito/peixe) & 2,62 & 8,53 & 11,05 & 17,27 & 38 \\
$* \mathrm{dp}$ & 2,56 & 8,93 & 8,36 & 14,48 & 14,14 \\
$* \mathrm{cv}(\%)$ & 0,97 & 1,04 & 0,75 & 0,83 & 0,37 \\
$* \mathrm{li}$ & 0 & 0 & 0 & 0 & 28 \\
$* \mathrm{ls}$ & 6 & 34 & 26 & 52 & 48 \\
\hline
\end{tabular}

*Dp - desvio-padrão, cv - coeficiente de variação, li - limite inferior, ls - limite superior.

Os espécimes de Hoplias malabaricus comercializados em São Bento-MA apresentavam potencial zoonótico, sugerindo risco para a população regional consumidora.A presença de pequenos crustáceos nas águas dos campos alagados da baixada maranhense contribui para infecção da traíras $(H$. malabaricus) pela larva Acanthella.

A presença de monogenéticos (Gyrodactyrus spp. e Dactylogyrius spp.), associada aos altos índices de prevalência observados, caracteriza um ambiente poluído, uma vez que estes parasitos atuam como indicador de alteração ambiental e têm sua população aumentada devido ao estresse do animal e às condições do meio em que vivem.

Palavras-chave: peixe, aspectos higiênicosanitários, endoparasitos

\begin{abstract}
The aim of this study was to verify the parasitologycal aspects of fish (Hoplias malabaricus), coming from São Bento city, Maranhão state. For this, 70 fish were used, verifying the parasitism indexes and coefficients. For the parasitological results nematodeos were identified, with zoonotical potential of the Contracaecum spp, Pseudoterranova spp, Eustrongylides spp; monogenetics Gyrodactirus spp, Dactylogirus spp and larva Acanthella of Acanthocephalo. Of the 70 (100\%) fishes, 64 (91.4\%) were sponged, with prevalence of $82.8 \%$ (58) for the nematodeo Contracaecum spp, 65.7\% (46), Pseudoterranova spp, 7.1\% (5), Eustrongylides spp, 71.4\% (50), monogenetics and larva Acanthella of Acantocephalo, respectively. A maior amplitude de intensidade (1-30), coeficiente de variação foi verificado para o nematódeo Pseudoterranova spp., a intensidade média $(7,3)$, índice de abundância $(6,7)$, coeficiente de dominância $(36,4)$ foi ao Contacaecum spp., todos os parasitos estavam no
\end{abstract}




\section{Rodrigues et al.}

mesentério da cavidade abdominal, encistados em forma larval.The largest intensity width (1-30), variation coefficient was verified for the nematodeo Pseudoterranova spp, the medium intensity $(7,3)$, abundance index (6,7), dominance coefficient $(36,4)$ it went to the Contacaecum spp, all of the parasites were in the mesenterio of the abdominal cavity, encysted in larval form. The results show that fish from this region presented unsatisfactory sanitary conditions in parasitological aspect and can expose consumers to the risks of foodborne illnesses.

Keywords: fish, hygienic and sanitary aspects, endoparasites

\section{REFERÊNCIAS}

AMATO, J.F.R.; BOEGER, W.A.; AMATO, S.B. Protocolo para laboratório: coleta e processamento de parasitas do pescado. Rio de Janeiro: Imprensa Universitária, UFRRJ, 1991. 81p.

ATLAS DO MARANHÃO. São Luís: GEPLAN, 2002. 44p.

BARROS, L.A.; OLIVEIRA, R.L.; MORAIS FILHO, J. et al. Análise do parasitismo por Contracaecum sp. e Eustrongylides sp. em cacharas, Pseudoplatystoma fasciatum (Linnaeus, 1766) (Pisces: Pimelodidae) provenientes do rio Cuiabá, Mato Grosso, Brasil. Rev. Bras. Ciênc. Vet., v.16, p.58-61, 2009.

EIRAS, J.C. Elementos de ictioparasitologia. Porto: Fundação Eng. Antonio de Almeida, 1994. 339p.

LUQUE, J.L.; POULIN, R. Use of fish as intermediate hosts by helminth parasites. Acta Parasitol., v.49, p.353-361, 2004

MARTINS, M.L.; SANTOS, R.S.; MARENGONI, N.G. et al. Seasonality of Eustrongylides sp. (Nematoda: dioctophymatidae) larvae in fishes from Paraná River, south-western Brazil. Bol. Inst. Pesca, v.35, p.29-37, 2009.
MULLER, M.I.; MADI, R.R.; UETA, M.T. Fauna helmíntica de Hoplias malabaricus (Bloch, 1794) nos tanques da fazenda das pedras. In: ASSOCIAÇÃO BRASILEIRA DE PATOLOGISTAS DE ORGANISMOS AQUÁTICOS, 8., 2004, Campinas. Anais... Laguna: EMBRAPOA, 2004. p.34.

NAKATANI, K.; AGOSTINHO, A.A.; BAUMGARTNER, G. et al. Ovos e larvas de peixes de água doce: desenvolvimento $\mathrm{e}$ manual de identificação. Maringá: UEM, 2001. 378p.

OKUMURA, M.P.M.; PÉREZ, A.C.A.; ESPÍNDOLA FILHO, A. Principais zoonoses parasitárias transmitidas por pescado: revisão. Rev. Educ. Cont. CRMV-SP., v.2, p.66-80, 1999.

OLSEN, O. Wilford. Parasitologia animal. Barcelona: Aedos, 1977. p.209-210.

PAVANELLI, G.C.; EIRAS, R.C.; TAKEMOTO, R.M. Doenças de peixes profilaxia, diagnóstico e tratamento. 3.ed. Maringá: Eduem, 2008. 311p. 